\title{
Progress toward third-order parametric down-conversion in optical fibers
}

\author{
Andrea Cavanna $\odot,{ }^{1,2, *}$ Jonas Hammer $\odot,{ }^{1,2}$ Cameron Okoth,,${ }^{1,2}$ Erasto Ortiz-Ricardo $\odot,{ }^{3}$ Hector Cruz-Ramirez, ${ }^{3}$ \\ Karina Garay-Palmett $\odot,{ }^{4}$ Alfred B. U’Ren, ${ }^{3}$ Michael H. Frosz $\odot,{ }^{1}$ Xin Jiang $\odot,{ }^{1}$ \\ Nicolas Y. Joly ${ }^{2},{ }^{2,1}$ and Maria V. Chekhova ${ }^{1,2}$ \\ ${ }^{1}$ Max Planck Institute for the Science of Light, Staudtstraße 2, 91058 Erlangen, Germany \\ ${ }^{2}$ University of Erlangen-Nürnberg, Staudtstraße 7/B2, 91058 Erlangen, Germany \\ ${ }^{3}$ Instituto de Ciencias Nucleares, Universidad Nacional Autónoma de México, Apartado Postal 70-543, 04510 Distrito Federal, Mexico \\ ${ }^{4}$ Departamento de Óptica, Centro de Investigación Científica y de Educación Superior de Ensenada, \\ Apartado Postal 2732, BC 22860 Ensenada, Mexico
}

(Received 14 October 2019; accepted 3 February 2020; published 30 March 2020)

\begin{abstract}
Optical fibers have been considered an optimal platform for third-order parametric down-conversion since they can potentially overcome the weak third-order nonlinearity by their long interaction length. Here we present, in the first part, a theoretical derivation for the conversion rate both in the case of spontaneous generation and in the presence of a seed beam. Then we review three types of optical fibers and we examine their properties in terms of conversion efficiency and practical feasibility.
\end{abstract}

DOI: 10.1103/PhysRevA.101.033840

\section{INTRODUCTION}

Optical fibers have proved to be extremely efficient platforms for generating nonclassical states [1-4]. While bulk materials and, more recently, waveguides are also good candidates for generating single- or two-photon states, the size and, more importantly, the length of both is limited technically. Optical fibers overcome this limitation, the only real constraints on their length being given by the optical loss and the homogeneity.

Direct generation of photon triplet states via the cubic interaction has been a long-standing goal in the field of quantum optics dating as far back as the 1980s [5-15]. The interest for this process, known as third-order parametric down-conversion (TOPDC), is driven by the fact that such an interaction leads to the direct generation of a non-Gaussian state [5]. It realizes a three-mode squeezing operator that differs greatly from the two-mode squeezing operator that leads to Gaussian squeezed states. Photon triplet states generated directly through the cubic interaction were observed only at microwave frequencies [16]. The photon triplet states generated so far at optical frequencies were mediated by the second-order susceptibility $[17,18]$. In the absence of postselection they do not display any non-Gaussian features.

In this work we estimate the efficiency of TOPDC in various fibers that have previously been suggested as promising platforms. Because the expected rates of three-photon emission are in most cases too small to be observed, we also

\footnotetext{
*Corresponding author: andrea.cavanna@mpl.mpg.de
}

Published by the American Physical Society under the terms of the Creative Commons Attribution 4.0 International license. Further distribution of this work must maintain attribution to the author(s) and the published article's title, journal citation, and DOI. Open access publication funded by the Max Planck Society. consider the case where TOPDC is seeded at the frequency of one of the three emitted photons. Seeding dramatically increases the rate of two-photon emission in the two remaining modes. Although the output state of these modes in this case is expected to be the same as for the usual two-photon spontaneous parametric down-conversion (SPDC) [19], seeding can be used to study the TOPDC spectral features, similar to the way stimulated emission tomography (SET) [20] is used to characterize SPDC.

\section{THEORY}

For a monochromatic pump, the rate of transitions from the vacuum state to nondegenerate three-photon state that occupies modes 1, 2, and 3 is given by the Fermi "golden rule,"

$$
\Gamma_{i \rightarrow f}=\frac{2 \pi}{\hbar^{2}}\left|\left\langle 1_{1}, 1_{2}, 1_{3}|\hat{H}| 0_{1}, 0_{2}, 0_{3}\right\rangle\right|^{2} \delta(\Delta \omega),
$$

where $\left\langle 1_{1}, 1_{2}, 1_{3}\right|$ is the final three-photon state, $\left|0_{1}, 0_{2}, 0_{3}\right\rangle$ is the initial vacuum state, $\hat{H}$ is the Hamiltonian, and $\Delta \omega=$ $\omega_{p}-\omega_{1}-\omega_{2}-\omega_{3}$. The subscript 1,2,3 denotes the state with frequency $\omega_{1,2,3}$ and propagation constant $\vec{\beta}_{1,2,3}$. Accounting for the possible transitions to several sets of modes as opposed to a single discrete three-mode state, we integrate Eq. (1) over a set of wave-vector intervals. The rate of transitions into this set of intervals is then

$$
\begin{aligned}
d R= & \frac{2 \pi}{\hbar^{2}}\left(\frac{L_{q}}{2 \pi}\right)^{3} \\
& \times\left|\left\langle 1_{1}, 1_{2}, 1_{3}|\hat{H}| 0_{1}, 0_{2}, 0_{3}\right\rangle\right|^{2} \delta(\Delta \omega) d \beta_{1} d \beta_{2} d \beta_{3},
\end{aligned}
$$

where $L_{q}$ is the quantization length. Using the dipole approximation and the third-order nonlinear response gives the Hamiltonian [19]

$$
\hat{H}=-24 \epsilon_{0} \chi_{\text {eff }}^{(3)} \int_{V_{\text {int }}} d^{3} \vec{r} E_{p}^{(+)} E_{1}^{(-)} E_{2}^{(-)} E_{3}^{(-)}+\text {H.c., }
$$


where $\chi_{\text {eff }}^{(3)}$ is the effective cubic susceptibility, $V_{\text {int }}$ is the volume of the cubic interaction, $\epsilon_{0}$ is the vacuum permittivity. The fields $E_{p}^{(+)}, E_{1}^{(-)}, E_{2}^{(-)}$, and $E_{3}^{(-)}$relate to the pump and modes 1,2 , and 3 , respectively, the $+(-)$ denote their positive (negative) frequency components. Please note that here we ignore terms for cross and self-phase modulation in the Hamiltonian and reintroduce them at a later stage as their effect on the phase matching is well documented [21]. We describe strong macroscopic fields such as the pump $(p)$ and later the seed $(s)$ classically,

$$
E_{p, s}^{(+)}=\mathcal{E}_{p, s} \widetilde{F}_{p, s}(x, y) e^{i \beta_{p, s} z},
$$

while the weak fields in modes 1, 2, 3 we describe using the quantum field operators

$$
\hat{E}_{n}^{(-)}=\widetilde{\mathcal{E}}_{n}^{*} \widetilde{F}_{n}^{*}(x, y) \hat{a}_{n}^{\dagger} e^{-i \beta_{n} z} .
$$

Here, $z$ is the propagation direction in the fiber, the subscript $n=p, 1,2,3$ denotes the field mode with frequency $\omega_{n}$, propagation constant $\beta_{n}$, refractive index $n\left(\omega_{n}\right)$, and group velocity $v_{g}\left(\omega_{n}\right), \hat{a}_{n}^{\dagger}$ is the photon creation operator. The normalized transverse field distribution

$$
\widetilde{F}_{n}(x, y)=\frac{F_{n}(x, y)}{\sqrt{\int\left|F_{n}(x, y)\right|^{2} d x d y}},
$$

where $F_{n}(x, y)$ is the unnormalized transverse field distribution [22]. The field amplitudes are given by

$$
\begin{aligned}
& \mathcal{E}_{p, s}=\sqrt{\frac{P_{p, s}}{2 c \epsilon_{0} n\left(\omega_{p, s}\right)}}, \\
& \tilde{\mathcal{E}}_{n}=i \sqrt{\frac{\hbar \omega_{n} v_{g}\left(\omega_{n}\right)}{2 \epsilon_{0} n\left(\omega_{n}\right) c L_{q}}},
\end{aligned}
$$

where $P_{p, s}$ is the power and $c$ is the speed of light.

\section{A. Unseeded TOPDC}

Substituting Eqs. (4) and (5) into the Hamiltonian (3) and integrating over the volume gives

$$
\hat{H}=-24 \epsilon_{0} \chi_{\mathrm{eff}}^{(3)} \frac{\mathcal{E}_{p} \widetilde{\mathcal{E}}_{1}^{*} \widetilde{\mathcal{E}}_{2}^{*} \widetilde{\mathcal{E}}_{3}^{*}}{A_{\text {eff }}} f(\Delta \beta) \hat{a}_{1}^{\dagger} \hat{a}_{2}^{\dagger} \hat{a}_{3}^{\dagger}+\text { H.c. }
$$

Here we have introduced the effective mode area as

$$
A_{\mathrm{eff}}=\left(\int \widetilde{F}_{p}(x, y) \widetilde{F}_{1}^{*}(x, y) \widetilde{F}_{2}^{*}(x, y) \widetilde{F}_{3}^{*}(x, y) d x d y\right)^{-1},
$$

and the phase matching function as

$$
f(\Delta \beta)=L \operatorname{sinc}\left(\frac{\Delta \beta L}{2}\right) \exp \left(i \frac{\Delta \beta L}{2}\right),
$$

where $L$ is the fiber length and the propagation constant mismatch is given by

$$
\Delta \beta=\beta_{p}-\beta_{1}-\beta_{2}-\beta_{3}-\beta_{N L} .
$$

We account for the cross and self-phase modulation terms, which we dropped from the Hamiltonian, by reintroducing a nonlinear momentum mismatch term $\beta_{N L}=\left[\gamma_{p}-2\left(\gamma_{p, 1}+\right.\right.$ $\left.\left.\gamma_{p, 2}+\gamma_{p, 3}\right)\right] P_{p}$, where $\gamma_{p}$ is the nonlinear coefficient for the pump self-phase modulation,

$$
\gamma_{p}=\frac{3 \chi^{(3)} \omega_{p}}{4 \epsilon_{0} c^{2} n_{p}^{2} A_{\mathrm{eff}}^{(p)}}
$$

and $\gamma_{p, n}$ is the nonlinear coefficient for cross-phase modulation,

$$
\gamma_{p, n}=\frac{3 \chi^{(3)} \omega_{n}}{4 \epsilon_{0} c^{2} n_{p} n_{n} A_{\mathrm{eff}}^{(p, n)}} .
$$

The effective mode area for the pump self-phase modulation is

$$
A_{\mathrm{eff}}^{(p)}=\left(\int\left|\widetilde{F}_{p}(x, y)\right|^{4} d x d y\right)^{-1},
$$

whereas for the cross phase modulation term the effective mode area between the pump and the photon triplet is

$$
A_{\mathrm{eff}}^{(p, n)}=\left(\int\left|\widetilde{F}_{p}(x, y)\right|^{2}\left|\widetilde{F}_{n}(x, y)\right|^{2} d x d y\right)^{-1} .
$$

It is worth noting that if the peak pump power is low, for example if one works in the continuous-wave (cw) regime, then the cross and self-modulation terms are negligible. Substituting Eq. (8) into Eq. (2) gives the following differential rate of triplet emission:

$$
\begin{aligned}
d R= & \frac{\hbar}{\pi^{2}} P_{p} \gamma_{1,2,3}^{2} \frac{\omega_{1} \omega_{2} \omega_{3}}{\omega_{p}^{2}} \\
& \times v_{g}\left(\omega_{1}\right) v_{g}\left(\omega_{2}\right) v_{g}\left(\omega_{3}\right)|f(\Delta \beta)|^{2} \delta(\Delta \omega) d \beta_{1} d \beta_{2} d \beta_{3},
\end{aligned}
$$

where the nonlinear coefficient is

$$
\gamma_{1,2,3}^{2}=\frac{9\left[\chi_{\mathrm{eff}}^{(3)}\right]^{2} \omega_{p}^{2}}{\epsilon_{0}^{2} c^{4} n_{p} n_{1} n_{2} n_{3} A_{\mathrm{eff}}^{2}} .
$$

Equation (16) can be rewritten in terms of frequency as opposed to propagation constant, using the relation for group velocity $\frac{d \beta_{n}}{d \omega_{n}}=\frac{1}{v_{g}\left(\omega_{n}\right)}$, as

$$
\begin{aligned}
d R\left(\omega_{1}, \omega_{2}, \omega_{3}\right)= & \frac{\hbar}{\pi^{2}} P_{p} \gamma_{1,2,3}^{2} \\
& \times \frac{\omega_{1} \omega_{2} \omega_{3}}{\omega_{p}^{2}}|f(\Delta \beta)|^{2} \delta(\Delta \omega) d \omega_{1} d \omega_{2} d \omega_{3} .
\end{aligned}
$$

Because the rate of unseeded TOPDC scales linearly with the pump power, it is more favorable to work in the cw regime. Working in the pulsed regime would lead to competing nonlinear processes that scale nonlinearly with the pump power. Light from such processes could saturate detectors or interfere with the spontaneous generation of triplets.

\section{B. Seeded TOPDC}

Stimulation of TOPDC requires a seed beam in one of the triplet modes, 1,2 , or 3 . In all the cases below we choose to replace mode 3 with the seed, which we will denote by the 

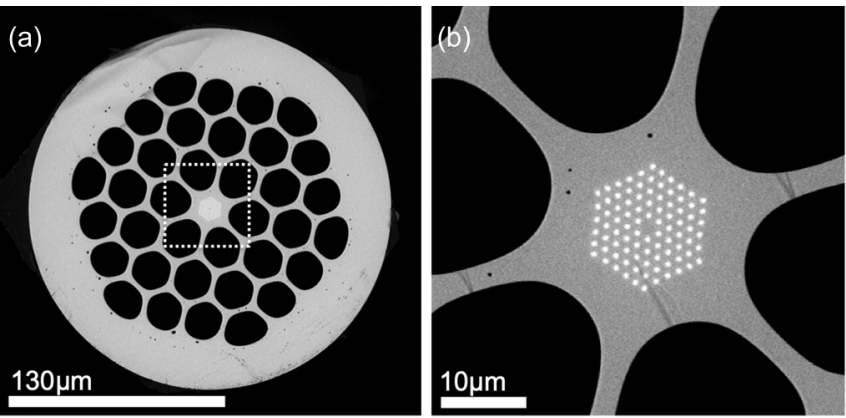

FIG. 1. SEM image of the hybrid fiber (a) and its inner core structure (b).

subscript $s$. If the seed beam is strong then it can be described by a classical field, hence the Hamiltonian in Eq. (8) can be rewritten as

$$
\hat{H}_{s}=-24 \epsilon_{0} \chi_{\mathrm{eff}}^{(3)} \frac{\mathcal{E}_{p} \mathcal{E}_{s}^{*} \widetilde{\mathcal{E}}_{1}^{*} \widetilde{\mathcal{E}}_{2}^{*}}{A_{\text {eff }}} f(\Delta \beta) \hat{a}_{1}^{\dagger} \hat{a}_{2}^{\dagger}+\text { c.c. }
$$

Here, the classical amplitude $\mathcal{E}_{s}^{*}$ of the seed is defined by Eq. (6) with $\omega_{s}=\omega_{3}$. From this it is clear that when a seed is present, the three-photon state is lost and instead a two-photon state is generated [19]. Since we now consider the product of two classical fields there is an advantage in using a pulsed source. If the seed and pump pulses are overlapped spatially and temporally, then their product averaged over time will introduce an enhancement factor equivalent to the inverse duty cycle of the laser.

In the pulsed regime the monochromatic approximation breaks down and the Fermi golden rule must be rewritten. Assuming a square pulse, the probability of a two-photon transition occurring over the pulse duration $t$ is

$$
\Pi_{i \rightarrow f}(t)=\frac{1}{\hbar^{2}}\left|\left\langle 1_{1}, 1_{2}\left|\hat{H}_{s}\right| 0_{1}, 0_{2}\right\rangle\right|^{2} \rho(\Delta \omega, t),
$$

where

$$
\rho(\Delta \omega, t)=t^{2} \operatorname{sinc}\left(\frac{\Delta \omega t}{2}\right)^{2} .
$$

Again integrating over a set of wave-vector intervals gives the differential number of transitions per pulse:

$$
d N=\frac{L_{q}^{2}}{4 \pi^{2} \hbar^{2}}\left|\left\langle 1_{1}, 1_{2}\left|\hat{H}_{s}\right| 0_{1}, 0_{2}\right\rangle\right|^{2} \rho(\Delta \omega, t) d \beta_{1} d \beta_{2} .
$$

Expanding out the quantum average gives

$$
\begin{aligned}
d N= & \frac{1}{\pi^{2}} P_{p} P_{s} \gamma_{1,2, s}^{2} \frac{\omega_{1} \omega_{2}}{\left(\widetilde{\omega}_{p}\right)^{2}} \\
& \times v_{g}\left(\omega_{1}\right) v_{g}\left(\omega_{2}\right)|f(\Delta \beta)|^{2} \rho(\Delta \omega, t) d \beta_{1} d \beta_{2},
\end{aligned}
$$

where the nonlinear interaction coefficient for the seeded process is

$$
\gamma_{1,2, s}^{2}=\frac{9\left[\chi_{\mathrm{eff}}^{(3)}\right]^{2}\left(\widetilde{\omega}_{p}\right)^{2}}{\epsilon_{0}^{2} c^{4} n_{p} n_{1} n_{2} n_{s} A_{\mathrm{eff}}^{2}}
$$

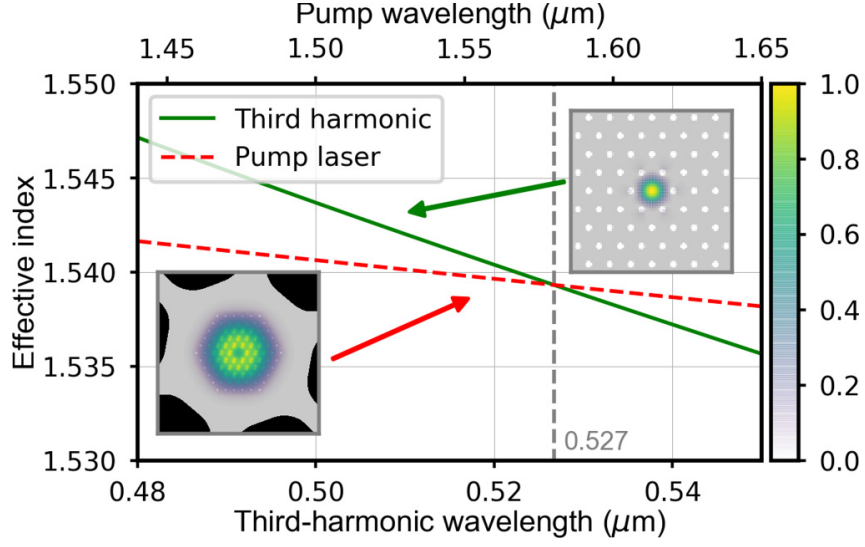

FIG. 2. Dispersion curves of the photonic band-gap mode and the infrared fundamental mode of the hybrid fiber. The insets show the calculated spatial distributions of the modes.

and $\widetilde{\omega}_{p}=\omega_{p}-\omega_{s}$. For practical reasons, we rewrite Eq. (23) in terms of frequency intervals, which yields

$$
\begin{aligned}
d N\left(\omega_{1}, \omega_{2}\right)= & \frac{1}{\pi^{2}} P_{p} P_{s} \gamma_{1,2, s}^{2} \\
& \times \frac{\omega_{1} \omega_{2}}{\left(\widetilde{\omega}_{p}\right)^{2}}|f(\Delta \beta)|^{2} \rho(\Delta \omega, t) d \omega_{1} d \omega_{2} .
\end{aligned}
$$

It is worth noting that $d N\left(\omega_{1}, \omega_{2}\right)$ and $d R\left(\omega_{1}, \omega_{2}, \omega_{3}\right)$ have different dimensionality, the former being the number of pairs emitted per pulse (dimensionless) and the latter being a rate of triplet emission $(\mathrm{Hz})$ in the $\mathrm{cw}$ regime.

\section{ESTIMATES AND EXPERIMENTAL EVIDENCE}

In order to ensure the efficient generation of photon triplets along the entire fiber length, phase matching has to be fulfilled; see Eq. (11). Normally this can be achieved by the so-called intermodal phase matching, where different fields propagate in the fiber in different spatial modes. In the case of triplet generation phase matching is usually found when the pump at $\omega_{p}$ is in a high-order mode while the three photons are in the fundamental mode. Coupling light into a high-order mode is quite challenging and usually not very efficient. Furthermore, there are no free parameters that allow one to tune the dispersion, therefore, for a standard optical

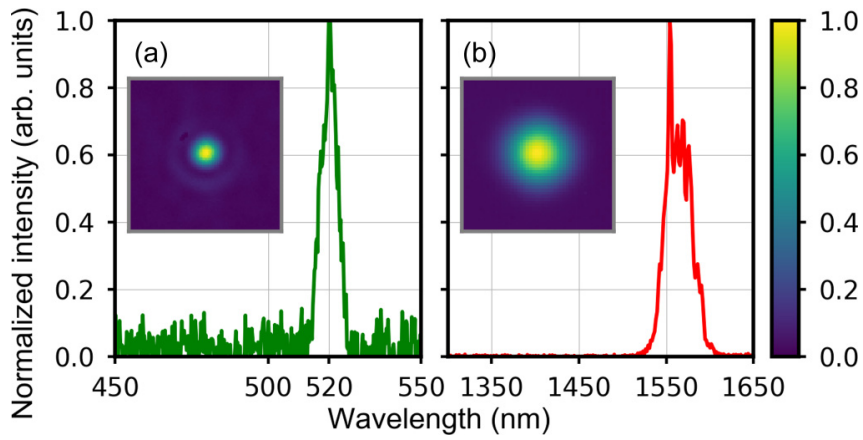

FIG. 3. Normalized spectra and near-field intensity distributions of the third harmonic (a) and the pump (b) for the hybrid fiber. The insets show the near field at the output of the fiber. 
TABLE I. Comparison of the expected triplet generation rate for different fiber types under realistic experimental conditions. The pump power of the tapered and the hybrid fibers is limited by the damage threshold, and for the hollow-core fiber by the high-order mode preparation. In the case of the solid-core fibers the length is limited by the fiber homogeneity and, in the case of hollow-core PCF, by the gas-cell dimensions.

\begin{tabular}{|c|c|c|c|c|c|c|c|}
\hline Fiber type & $\begin{array}{c}\text { Pump power } \\
\left(P_{p}\right)\end{array}$ & $\begin{array}{c}\chi_{\mathrm{eff}}^{(3)} \\
\left(10^{-22} \mathrm{~m}^{2} \mathrm{~V}^{-2}\right)\end{array}$ & $\begin{array}{c}\text { Pump } \\
\text { wavelength } \\
(\mu \mathrm{m})\end{array}$ & $\begin{array}{l}\text { Effective } \\
\text { area }\left(A_{\text {eff }}\right) \\
\left(\mu \mathrm{m}^{2}\right)\end{array}$ & $\begin{array}{l}\text { Length }(L) \\
\quad(\mathrm{cm})\end{array}$ & $\begin{array}{c}\text { Detect. } \\
\text { bandwidth } \\
(\Delta \lambda)(\mathrm{nm})\end{array}$ & $\begin{array}{c}\text { Triplet rate } \\
(\mathrm{Hz})\end{array}$ \\
\hline Hybrid core & $100 \mathrm{~mW}$ & 11.5 & 0.526865 & 218 & 10 & 150 & 11 \\
\hline Hollow core & $200 \mathrm{~mW}$ & 0.043 & 0.532 & 19200 & 100 & 150 & $5.5 \times 10^{-6}$ \\
\hline Tapered & $20 \mathrm{~mW}$ & 2.5 & 0.532 & 7.89 & 10 & 150 & 3.2 \\
\hline
\end{tabular}

fiber the phase matching frequencies are fixed. To overcome these two problems we investigated different types of optical fibers. The first one is a hybrid fiber that, due to the use of different guidance mechanisms, allows the phase matching between single-lobed modes. Not only does this simplify the coupling, but it also increases the overlap between the pump and the generated fields. The second one is a hollowcore fiber filled with xenon gas, which allows tunable phase matching by changing the gas pressure. Finally, we consider a tapered fiber, which has a high overlap between the two phase matching modes due to their high confinement and whose dispersion can be tuned by changing the gas pressure of the environment [23].

\section{A. Hybrid fiber}

The first fiber that we present is a solid core microstructured fiber with a double core structure [13]. This fiber was designed to circumvent the problem of coupling the pump beam into a high-order mode by compensating the phase mismatch using modes of different size, ideally both fundamental. This comes from the fact that the propagation constant of a mode at a fixed wavelength can be decreased by just reducing the mode diameter. The fiber has a photonic band-gap (PBG) structure that guides the visible mode, surrounded by hollow channels. The infrared mode is guided by total internal reflection, the entire PBG structure acts as the core, and the surrounding glass, together with the hollow channels, as the cladding. The inner PBG structure contains rods made of high refractive index lead-silicate glass (Schott SF6) embedded in low refractive index glass (Schott LLF1); see Fig. 1 for the scanning electron micrograph (SEM) of the fiber. The central rod of SF6 glass is replaced by one of LLF1 in order to create a defect, that opens up a band gap, allowing guiding in the core. By carefully tuning the diameter of the rods and the distance
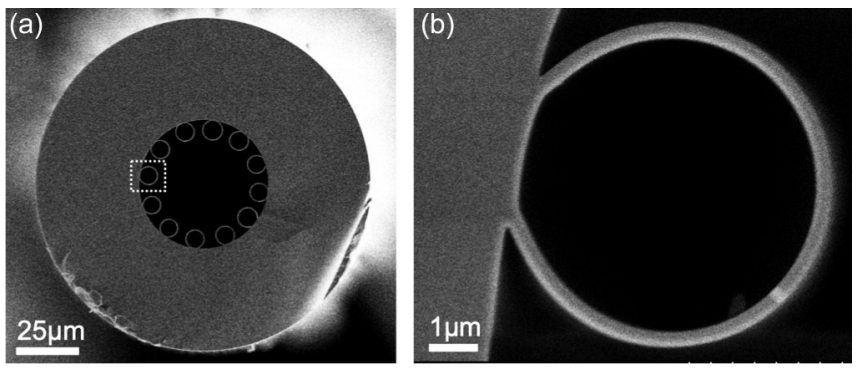

FIG. 4. SEM picture of the hollow-core structure (a) with a detailed view of a capillary (b). between them (the pitch), it is possible to create a band gap that confines light at a particular frequency [24]. In our case, the target wavelength is $532 \mathrm{~nm}$. For our fiber we choose a diameter of about $380 \mathrm{~nm}$ for the SF6 glass rods and a pitch of $1.05 \mu \mathrm{m}$. The rods are arranged in a hexagonal geometry with five concentric rings. On the one hand, increasing the number of rings will decrease the guidance losses of the visible mode but on the other hand, the dimensions of the PBG structure define the infrared mode size and therefore its dispersion. Figure 2 shows the dispersion and the intensity distributions of guided modes simulated with the finite-element model (FEM) [13].

Photon triplet generation can be seen as the reverse process of third-harmonic generation (THG), with which it shares, among many other features, the phase matching conditions. We therefore used THG to test the phase matching for the manufactured fiber. Due to the small dimensions it is difficult to precisely control the fiber parameters and achieve phase matching at exactly $532 \mathrm{~nm}$. Therefore we used an optical parametric generator (OPG) pumped with the second harmonic of a Nd:YAG laser $(532 \mathrm{~nm})$ with 20 ps pulse duration and $1 \mathrm{kHz}$ repetition rate [25]. The output of the OPG was tunable over more than $200 \mathrm{~nm}$ with about $10 \mathrm{~mW}$ average power. Using this source we generated third-harmonic radiation in the optical fiber. The phase matching wavelength was found at $520 \mathrm{~nm}$ and the near field intensity distribution of the mode was measured; see Fig. 3.

Starting from the mode electric fields, obtained with the FEM simulations, we calculated an effective area of $A_{\mathrm{eff}}^{(p, n)}=$ $218 \mu \mathrm{m}^{2}$. We considered the effective third-order susceptibility of SF6 glass $\chi^{(3)}=1.15 \times 10^{-21} \mathrm{~m}^{2} \mathrm{~V}^{-2}$ [26]. Using

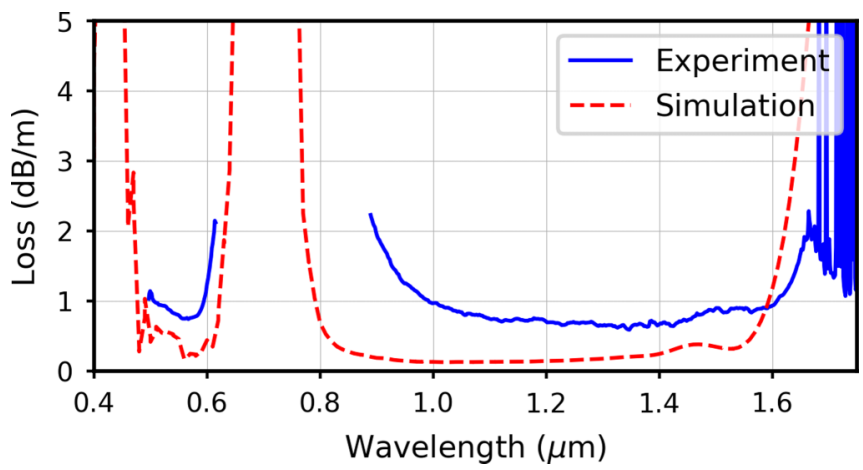

FIG. 5. The calculated (red dashed line) and measured (blue continuous line) loss for the single-ring PCF designed for the photon triplet generation. 


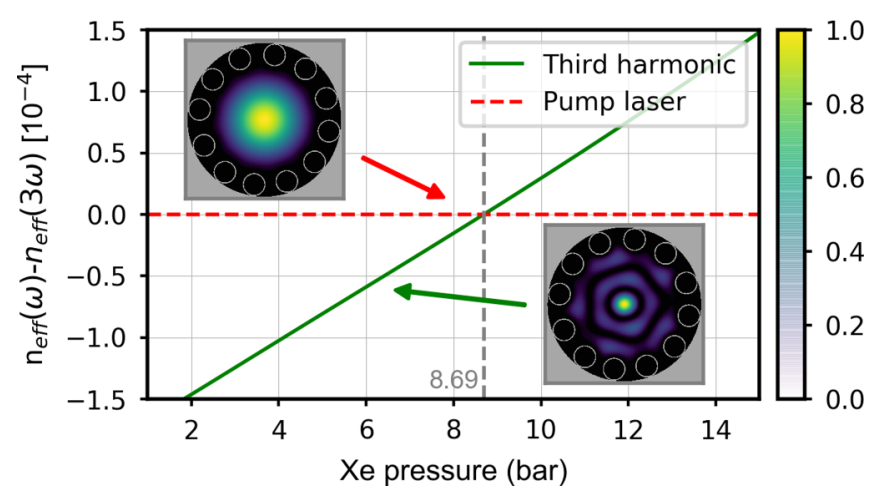

FIG. 6. Difference between the refractive indices for 532 and $1596 \mathrm{~nm}$ vs the Xe pressure in a single-ring PCF. The insets show the simulated intensity distributions of the modes. The insets show the near field at the output of the fiber.

Eq. (18) and assuming a pump power of $100 \mathrm{~mW}$ (cw), a length of $10 \mathrm{~cm}$, and a detection bandwidth of $150 \mathrm{~nm}$, we obtained an expected spontaneous triplet generation rate of $11 \mathrm{~Hz}$ (Table I).

\section{B. Gas-filled hollow-core fiber}

The second fiber that we present is a gas-filled hollowcore photonic-crystal fiber (PCF). Hollow-core fibers have the advantage that they allow tunable phase matching by changing the pressure of the filling gas. For our experiment we choose to use xenon due to its high nonlinearity [27] and the very low reactivity. There are several geometries of hollow-core fibers; the one that we consider in this paper is known as single-ring fiber [28,29]. It consists of a ring of capillaries attached to the inner surface of a glass tube; see Fig. 4(a). Light is confined in the core region by means of antiresonant reflection at the core-cladding interface. The wall thickness of the glass capillaries defines the resonant frequencies at which the light is not guided. The dimensions of the capillaries determine the modal guidance of the fiber. The light is guided in the core only if there is no coupling with the capillary modes [30]. For triplet generation we require minimum guidance losses for
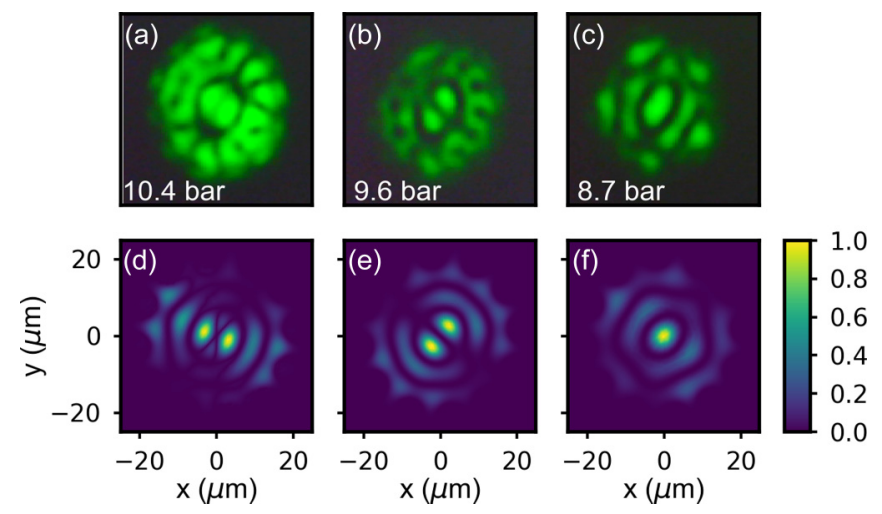

FIG. 7. Phase matched third-harmonic high-order modes generated by changing the xenon pressure inside the gas chamber [from (a) to (c)]. Simulated Poynting vector of the guided modes [from (d) to (f)].

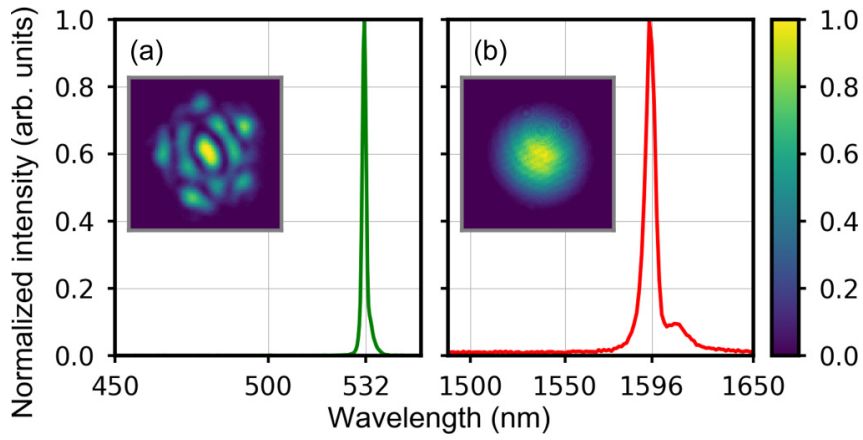

FIG. 8. Normalized spectra and measured near-field intensity distributions of the TH (a) and the pump (b) for Xe-filled PCF at 8.7 bars.

infrared light around $1600 \mathrm{~nm}$ in the fundamental mode and at $532 \mathrm{~nm}$ for the phase-matched high-order mode. Depending on the core diameter, the phase matching can be achieved at different gas pressures [31]. A smaller core size usually implies higher guidance losses but at the same time also a higher gas pressure at which phase matching is achieved and therefore higher nonlinearity. The designed fiber has a core diameter of $38.7 \mu \mathrm{m}$ surrounded by a ring of 12 capillaries, each with a diameter of $7.1 \mu \mathrm{m}$. For this configuration there is no coupling between the visible high-order mode and the capillaries' modes at the same frequency.

Using the formula given in Refs. [32,33], it is possible to estimate the resonance frequencies for given parameters of the fiber. The glass thickness of the capillaries was chosen to be $350 \mathrm{~nm}$ in order to set the resonant frequencies far from the region of interest. The drawn fiber, with the corresponding glass thickness, has relatively low losses both at $532 \mathrm{~nm}$ and around $1596 \mathrm{~nm}$. Figure 5 shows that, in agreement with the calculation (red line), the measured (blue line) loss in the ranges of interest does not exceed $1 \mathrm{~dB} / \mathrm{m}$.

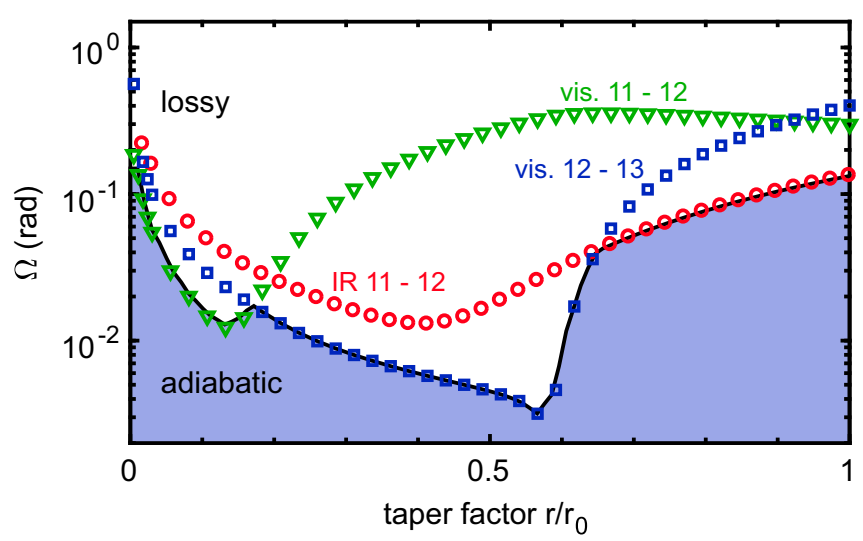

FIG. 9. Maximal angle allowing an adiabatic transition for $\mathrm{HE}_{11}$ and $\mathrm{HE}_{12}$, with the pump in the visible and the generated signal in the IR. The taper should be designed such that the local angle always lies within the shaded area to ensure adiabaticity. The red dots correspond to the maximum angle to avoid coupling from the IR $\mathrm{HE}_{11}$ to the next relevant higher-order mode $\mathrm{HE}_{12}$. The green triangles (blue squares) are the criterion for the visible light to avoid coupling between the $\mathrm{HE}_{12}$ and the next relevant lower- (higher-) modes. 


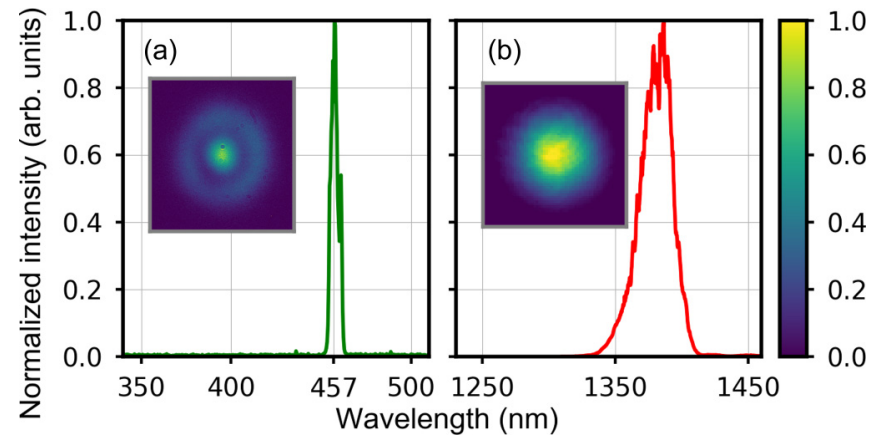

FIG. 10. Normalized spectra and measured near-field intensity distributions of the TH (a) and the pump (b) for tapered single-mode fiber in vacuum. The insets show the Poynting vector distributions at the output of the fiber.

Using a FEM model of the fiber, we simulated the guided modes and their dispersion as a function of the Xe pressure; see Fig. 6. The phase matching was found at 8.7 bars.

In order to verify the phase matching we coupled the output of our OPG tuned at $1596 \mathrm{~nm}$ into the fiber core and observed the THG. To reduce the bandwidth of the pump beam we used a cw seed beam at $1596 \mathrm{~nm}$ in the OPG. The fiber was mounted in a gas cell with a Xe pressure of about 15 bars. By gradually reducing the gas pressure we achieved the THG for different high-order modes at $532 \mathrm{~nm}$; see Fig. 7. Our target mode, i.e., the one with the lowest possible order and therefore with the lowest effective area, was achieved at 8.7 bars. Figure 8 shows the recorded spectra and the intensity distributions for the target mode at $532 \mathrm{~nm}$ and the fundamental IR mode.

In order to estimate the level of luminescence occurring in the gas-filled hollow-core experiment, we pumped the fiber with a cw pump beam at $532 \mathrm{~nm}$. We measured the level of output signal in the spectral range of TOPDC, making use of a self-made monochromator [23]. In the case of solid-core fibers, the material absorption in the visible yields a broadband emission of luminescence, which can extend over the spectral range of TOPDC, hampering if not totally preventing its measurement [34]. In the case of hollow-core fibers, the

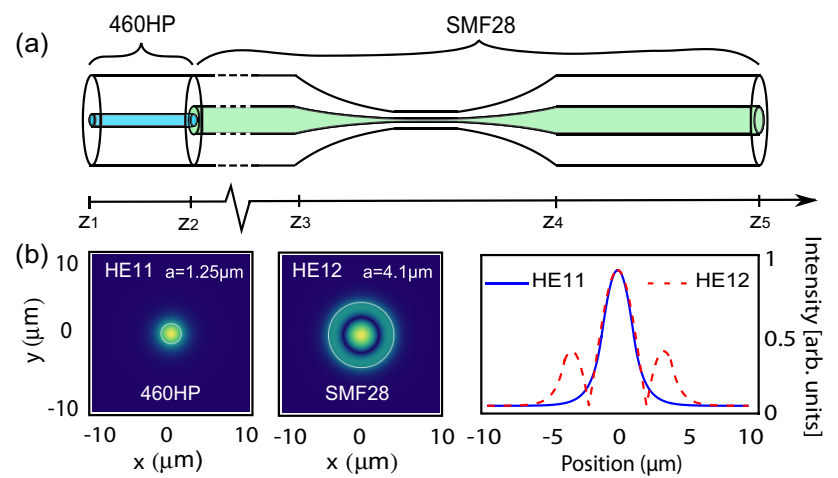

FIG. 11. (a) Proposed device based on 460HP and SMF28 fibers spliced together, with a taper in the SMF28 stretch. (b) $\mathrm{HE}_{11}$ mode propagating in the $460 \mathrm{HP}$ fiber, $\mathrm{HE}_{12}$ mode propagating in the SMF28 fiber, and diametrical section of both modes.

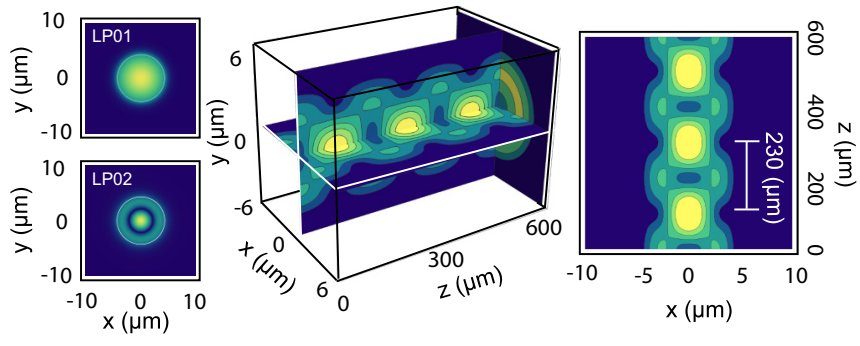

FIG. 12. Left: $\mathrm{HE}_{11}$ and $\mathrm{HE}_{12}$ modes in the SMF28 fiber. Middle: Resulting intensity pattern in the $z y, x z$, and $x y$ planes. Right: Resulting intensity pattern in the $x z$ plane.

measurement does not show any change in the dark counts, even at $200 \mathrm{~mW}$ pump power. This confirms that the number of photons generated is below $10 \mathrm{~Hz}$, which is the sensitivity of our apparatus.

The third-order nonlinearity of xenon can be estimated from Refs. [27,35] $\chi_{0}^{(3)}=6.4 \times 10^{-26} \mathrm{~m}^{2} \mathrm{~V}^{-2}$, where the subscript 0 indicates that the susceptibility was measured at a pressure of 1 bar and at room temperature. The effective nonlinearity is proportional to the gas pressure, therefore at 8.7 bars, the susceptibility is about nine times larger than $\chi_{0}^{(3)}$. It is worth noting that by reducing the core diameter and improving the fiber design (to reduce the confinement losses), not only will the effective area decrease but also the phase matching will move to higher pressure where the nonlinearity is higher. Due to the relatively low third-order nonlinearity of gases compared to solids, in the hollow-core PCF the spontaneous triplet generation rate is on the order of $10^{-5} \mathrm{~Hz}$ for pumping $1 \mathrm{~m}$ of fiber with $200 \mathrm{~mW}$ (Table I). Nevertheless, hollow-core PCFs are very suitable for seeded generation, since they can guide high laser power without being damaged. The only restrictions are the preparation and the coupling efficiency of the high-order pump mode.

\section{Tapered fiber}

The final fiber under consideration as a platform for photon-triplet generation is a tapered optical fiber. Fiber tapers are manufactured by stretching a conventional step-index fiber (SMF28) over a scanning oxybutane flame [36]. Controlling the pulling speed and scanning range allows for a precise control over the taper parameters. Decreasing the diameter of the

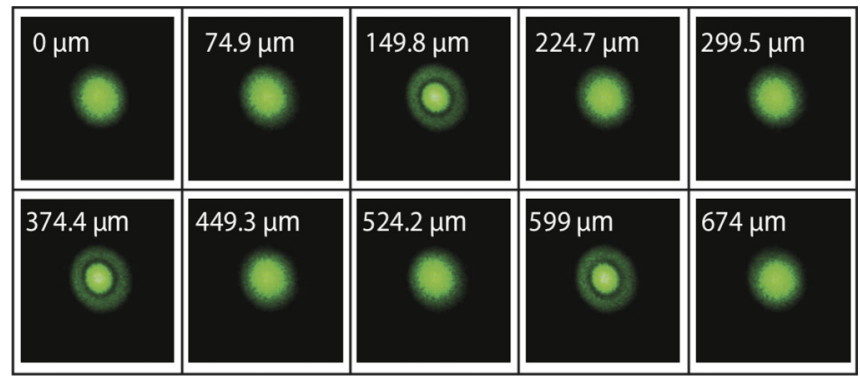

FIG. 13. Imaged intensity pattern at the output of the fiber during taper fabrication, obtained from a video recorded by a chargecoupled device camera, with a 90-ms time between frames, corresponding to a $\sim 75 \mu \mathrm{m}$ taper length differential between frames. 
TABLE II. Comparison of the expected pair generation rate for different fiber types in the case of a single seed. The pump and the seed powers of all the fibers are chosen to limit the generation of photon pairs to allow coincidence measurements. For the calculation of the number of generated photon pairs we considered a pulse duration of $20 \mathrm{ps}$.

\begin{tabular}{lccccc}
\hline \hline Fiber type & Pump pulse energy & Seed wavelength $(\mu \mathrm{m})$ & Seed pulse energy & Detect. bandwidth $(\Delta \lambda)(\mathrm{nm})$ & Number of pairs $($ per pulse) \\
\hline Hybrid core & $5 \mathrm{~nJ}$ & 1.67 & $1 \mathrm{~nJ}$ & 60 & 0.48 \\
Hollow core & $200 \mu \mathrm{J}$ & 1.605 & $10 \mu \mathrm{J}$ & 200 & 0.50 \\
Tapered & $1 \mathrm{~nJ}$ & 1.6412 & $0.1 \mathrm{~nJ}$ & 50 & 0.52 \\
\hline \hline
\end{tabular}

fiber increases its effective nonlinearity, as well as drastically changing the waveguide contribution to the dispersion. The fiber tapers of interest here have a submicron waist diameter. At this diameter the initial fiber core is reduced so much that it does not play any significant role in the guiding mechanism, and the dispersion of the taper waist is given by the dispersion of a silica rod with the corresponding diameter in vacuum [37]. For triplet generation, the use of tapered optical fiber requires intermodal phase matching similar to the case of gas-filled hollow-core fibers.

As shown by Corona et al. [11], the most favorable case occurs when the visible pump light is guided in the $\mathrm{HE}_{12}$ mode and the generated photon triplet state is in the fundamental mode of the fiber. It is essential to ensure an adiabatic transition for both spatial modes involved in the generation process. Therefore, the transition profile of the taper must be designed very carefully. In particular, the local transition angle $\Omega(z)$ must remain small enough to avoid coupling between the fiber modes. The maximum angle allowed for adiabatic mode conversion between the untapered fiber and the taper waist is given by [38]

$$
\Omega=\frac{\rho}{2 \pi}\left(\beta_{i}-\beta_{i \pm 1}\right)
$$

where $\rho$ is the local core radius of the taper, $\beta_{i}(z)$ is the local wave number of the $i$ th mode to couple into the waist taper and $\beta_{i+1}$ is the closest mode, to which coupling should be avoided. Here, we used a finite-difference eigenmode solver to compute the wave number for the $\mathrm{HE}_{11}$ and $\mathrm{HE}_{12}$ for the IR and the same two modes plus the $\mathrm{HE}_{13}$ in the visible (Fig. 9). In this calculation the $\mathrm{HE}_{12, \mathrm{IR}}$ and $\mathrm{HE}_{13 \text {,vis }}$ are guided at the cladding-air interface at all places along the transition, while the other modes are guided in the core of the untapered fiber and evolve into cladding modes along the transition. Using Eq. (26) we can calculate the three different curves in Fig. 9 corresponding to the adiabatic angles that avoid coupling between $\mathrm{HE}_{11}$ and $\mathrm{HE}_{12}$ in both the visible and the IR, as well as avoiding coupling between $\mathrm{HE}_{12}$ and $\mathrm{HE}_{13}$ in the visible. The actual transition should always lie below all three curves.

Pumping at $532 \mathrm{~nm}$, the diameter of the tapered waist should be $790 \mathrm{~nm}$ for degenerate photon triplet generation. In this case, the effective area $A_{\text {eff }}=7.9 \mu \mathrm{m}^{2}$ and using $\chi_{\text {eff }}^{(3)}=10^{-22} \mathrm{~m}^{2} \mathrm{~V}^{-2}$ for silica we can evaluate a generation rate as high as $3.2 \mathrm{~Hz}$ for a $10-\mathrm{cm}$-long taper with a pump of $20 \mathrm{~mW}$ (Table I).

It is important to note that the exact value of the diameter is extremely critical. With the same pumping condition but a tapered waist of $791 \mathrm{~nm}$ the phase matching is no longer degenerate and the emission bandwidth is $130 \mathrm{~nm}$ (see the Appendix). The same effect can be obtained by changing the pump wavelength by about $0.5 \mathrm{~nm}$. Such stringent fabrication tolerance cannot be met in practice. We can however circumvent this difficulty by encapsulating the tapered fiber inside a gas cell with an adjustable pressure [23].

Similarly to the other fibers, third-harmonic generation was measured in the fiber taper. The fiber was pumped with a tunable laser that had a $0.25-\mathrm{MHz}$ repetition rate, $160-\mathrm{fs}$ pulse duration, and a central wavelength at $1375 \mathrm{~nm}$; see Fig. 10. The third harmonic was generated at $457 \mathrm{~nm}$ in the expected $\mathrm{HE}_{12}$ guided mode.

One of the challenges that we face when employing tapered fibers for TOPDC is the difficulty of preferentially coupling the pump, at $532 \mathrm{~nm}$, to the $\mathrm{HE}_{12}$ mode. We have explored a solution which involves the use of two different types of fiber, 460HP and SMF28 spliced together, as shown schematically in Fig. 11(a), with the tapered region appearing along the SMF28 stretch of fiber. While the 460HP fiber is characterized by a core diameter of $2.5 \mu \mathrm{m}$ and is single-mode at $532 \mathrm{~nm}$, the SMF28 fiber with a core diameter of $8.2 \mu \mathrm{m}$ is multimode at this wavelength (but single mode at the TOPDC center wavelength of $1596 \mathrm{~nm}$ ).

The concept that we exploit is that the fundamental mode $\mathrm{HE}_{11}$ at $532 \mathrm{~nm}$ propagating in the $460 \mathrm{HP}$ fiber excites preferentially, upon arrival to the intrafiber interface, the $\mathrm{HE}_{12}$
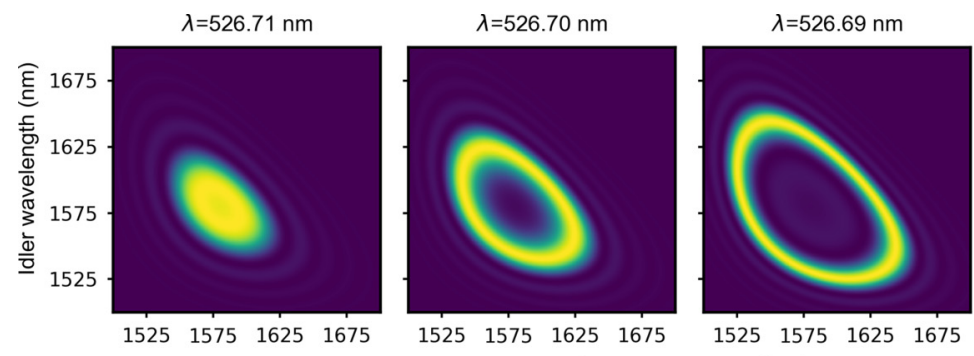

Signal wavelength $(\mathrm{nm})$

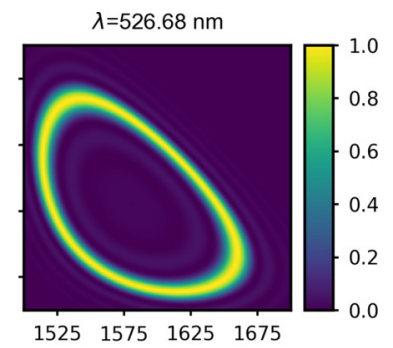

FIG. 14. The spectral density $S\left(\omega_{1}, \omega_{2}\right)$ for the hybrid-core fiber at different pump frequencies. 
mode in the SMF28 fiber. This occurs because (i) the mode field diameter of the $\mathrm{HE}_{11}$ mode in the $460 \mathrm{HP}$ fiber is very similar to the diameter of the internal lobe of the $\mathrm{HE}_{12}$ mode in the SMF28 fiber, and (ii) the effective index of refraction of the incoming $\mathrm{HE}_{11}$ mode and that of the excited $\mathrm{HE}_{12}$ mode are very similar, differing only by $\sim 0.001$. In Fig. 11(b) we plot the simulated incoming $\mathrm{HE}_{11}$ mode, as well as the excited $\mathrm{HE}_{12}$ mode, along with a diametrical section of these two modes overlapped in a single plot making it clear that the modes are remarkably similar within the internal lobe. This leads to an unusually high mode overlap between the incoming and excited modes (at approximately the same level as the overlap between the fundamental modes in the two fibers). Note that the SMF28 can support higher-order modes but the overlap of these modes with $\mathrm{HE}_{11}$ from the 460HP drops dramatically.

In the SMF28, the presence of the $\mathrm{HE}_{11}$ and $\mathrm{HE}_{12}$ modes [transverse amplitudes $E_{1}(x, y)$ and $E_{2}(x, y)$, and propagation constants $\beta_{1}$ and $\beta_{2}$ respectively] leads to a mode beating with a period $2 \pi /\left(\beta_{1}-\beta_{2}\right)=230 \mu \mathrm{m}$. In Fig. 12 we show the evolution of the intensity pattern along the fiber resulting from the beating of the fundamental $\mathrm{HE}_{11}$ and higher-order mode $\mathrm{HE}_{12}$.

We fabricated a device as described above, with 460HP and SMF28 fibers spliced together. The SMF28 is then tapered down to $\sim 0.8 \mu \mathrm{m}$ diameter. As the taper is made its length increases. During this process it is then possible to observe, in real time, the evolution of the beating pattern between the two modes involved as a function of length by monitoring the output of the device. Figure 13 shows the evolution of the intensity of the near field at the output of the SMF28 as a function of the added length. This is consistent with the simulated beating period of $230 \mu \mathrm{m}$, confirming the considered approach.

\section{Seeding}

Although seeding third-order parametric down-conversion reduces the generated state from a three-photon to a twophoton state, it does dramatically increase the emission rate probability. While the hybrid and tapered fibers have measurable spontaneous three-photon emission rates, due to the poor overlap and low cubic susceptibility the hollow-core fiber does not (see Table I). Hence to observe any effect in the hollow-core fiber, stimulating one of the three photons is a necessity. Despite losing the three-photon correlations, seeding can still lead to information about the spontaneous three-photon state using stimulated emission tomography. Moving to the seeded emission probabilities it is clear that one can observe photon pairs for each fiber type. Here we assume a pulsed laser with a 20 -ps pulse duration and $1-\mathrm{kHz}$ repetition rate, hence working with single-photon detectors the number of photons detected for the hybrid and tapered fibers would be limited by the repetition rate. In order to perform a proper correlation measurement, the pump or the seed power has to be reduced to generate less then a pair per pulse. The estimated conversion probabilities with seeding are given in Table II.

\section{CONCLUSION}

We presented three different types of fibers and estimated their potential viability as platforms on which to generate photon triplet states via a direct cubic interaction. We checked this for both the spontaneous and seeded regimes. The hybrid and tapered fibers proved to be the best fibers from which to observe photon triplets. However the former requires a tunable pump laser to satisfy phase matching due to its fixed geometry. Despite this drawback, the main advantage the hybrid fiber has over the other two designs is the ability to guide both the pump and triplets in a nearly Gaussian mode simultaneously. In contrast to the hybrid fiber, one can tune the phase matching in the the tapered fiber without changing the pump wavelength. This can be done by placing the tapered fiber into a gas with tunable pressure. In addition, with the system we propose, it is possible to launch a high-order mode into the tapered fiber starting from a nearly Gaussian mode. Currently the design of the hollow-core fiber gives a very small conversion efficiency. However this can be improved by reducing the core size. By reducing the core diameter by only a factor of 2 , the conversion efficiency can be improved by around two orders of magnitude. In this case, a hollow-core fiber, with its high damage threshold and the pressure-dependent phase matching, is a good candidate for seeding experiments.

\section{APPENDIX: SPECTRAL DENSITY OF THREE-PHOTON EMISSION}

The expression for $d R\left(\omega_{1}, \omega_{2}, \omega_{3}\right)$ in Eq. (18) can be simplified to a function of two variables by integrating $\omega_{3}$ over the delta function $\delta(\Delta \omega)$. Due to energy conservation this fixes the frequency of $\omega_{3}=\omega_{p}-\omega_{1}-\omega_{2}$. Thus we can
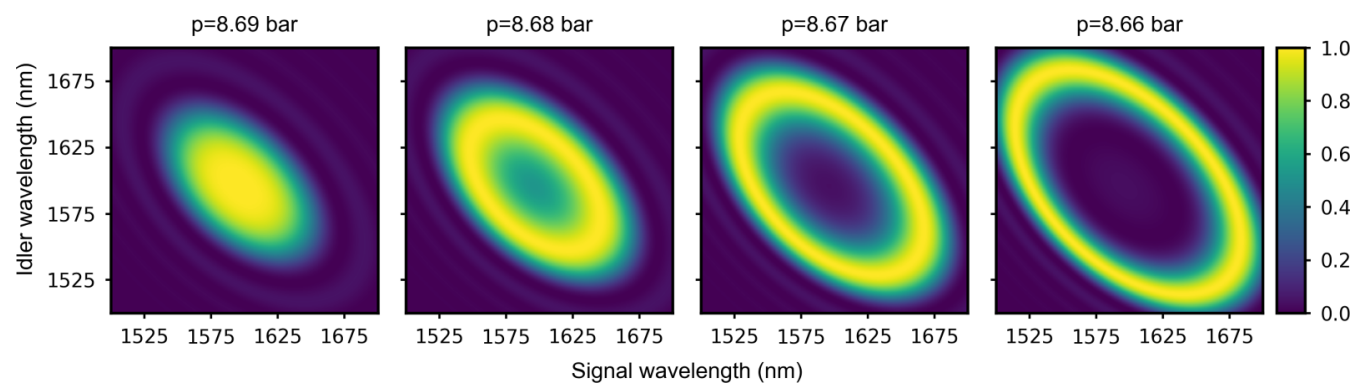

FIG. 15. The spectral density $S\left(\omega_{1}, \omega_{2}\right)$ for the hollow-core fiber at different xenon pressures. 


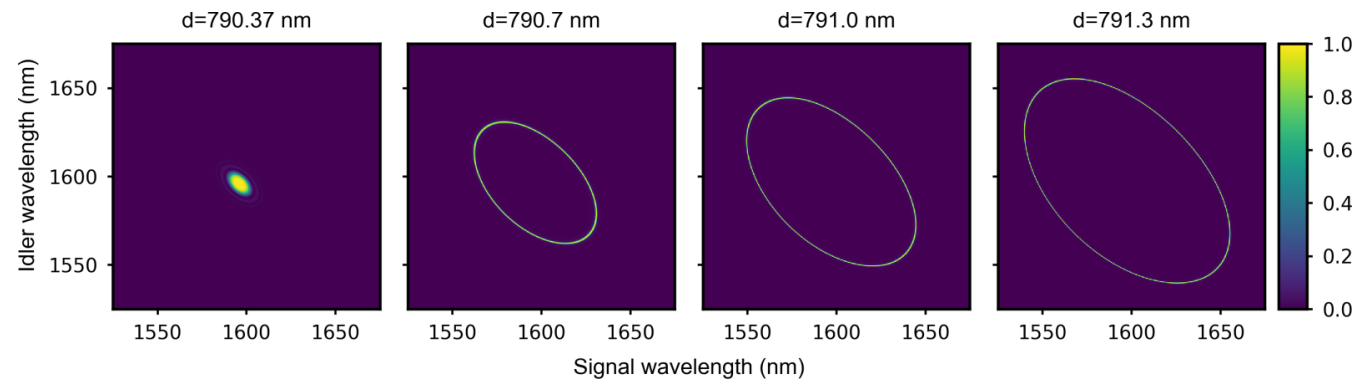

FIG. 16. The spectral density $S\left(\omega_{1}, \omega_{2}\right)$ for the tapered fiber at different taper diameters.

rewrite Eq. (18) as

$$
\begin{aligned}
d R\left(\omega_{1}, \omega_{2}\right)= & \frac{\hbar}{2 \pi^{2}} P_{p} \gamma_{1,2,3}^{2} \\
& \times \frac{\omega_{1} \omega_{2}\left(\omega_{p}-\omega_{1}-\omega_{2}\right)}{\omega_{p}^{2}}|f(\Delta \beta)|^{2} d \omega_{1} d \omega_{2} .
\end{aligned}
$$

Plotting $S\left(\omega_{1}, \omega_{2}\right)=\frac{d R\left(\omega_{1}, \omega_{2}\right)}{d \omega_{1} d \omega_{2}}$ gives the spectral density of the three-photon emission.

Figure 14 shows the spectral density for the hybrid fiber for the parameters shown in Table I. Varying the pump wavelength by only $0.01 \mathrm{~nm}$, one changes the spectral density vastly. This is due to the extremely different dispersion properties of the inner and outer core. In addition the spectral density is very broad, ranging over almost $150 \mathrm{~nm}$. A combination of these two attributes makes it critical for the pump to be narrow-band enough to minimize the collection bandwidth and increase the signal-to-noise ratio.

Figure 15 shows the spectral density for the hollow-core fiber filled with xenon gas and pumped at $532 \mathrm{~nm}$. By reducing the gas pressure it is possible to pass from degenerate to nondegenerate phase matching. Although the spectral width has a strong dependence on the pressure, this can be easily and precisely controlled with standard equipment.

Finally, in Fig. 16 the spectral density for the tapered fiber is shown. The tapered region is highly dispersive due to the high confinement and increasing mode leakage into the ambient environment at longer wavelengths. This leads to a very narrow emission bandwidth. Although it means that the phase matching is critical, it is an advantage to work with the narrow spectrum as it means that the full emission spectrum can be collected.
[1] H. Takesue and K. Inoue, Generation of polarization-entangled photon pairs and violation of bell's inequality using spontaneous four-wave mixing in a fiber loop, Phys. Rev. A 70, 031802(R) (2004).

[2] J. G. Rarity, J. Fulconis, J. Duligall, W. J. Wadsworth, and P. S. J. Russell, Photonic crystal fiber source of correlated photon pairs, Opt. Express 13, 534 (2005).

[3] X. Li, P. L. Voss, J. E. Sharping, and P. Kumar, OpticalFiber Source of Polarization-Entangled Photons in the $1550 \mathrm{~nm}$ Telecom Band, Phys. Rev. Lett. 94, 053601 (2005).

[4] J. Fan, A. Migdall, and L. J. Wang, Efficient generation of correlated photon pairs in a microstructure fiber, Opt. Lett. 30, 3368 (2005).

[5] S. L. Braunstein and R. I. McLachlan, Generalized squeezing, Phys. Rev. A 35, 1659 (1987).

[6] P. V. Elyutin and D. N. Klyshko, Three-photon squeezing: Exploding solutions and possible experiments, Phys. Lett. A 149, 241 (1990).

[7] T. Felbinger, S. Schiller, and J. Mlynek, Oscillation and Generation of Nonclassical States in Three-Photon Down-Conversion, Phys. Rev. Lett. 80, 492 (1998).

[8] J. Douady and B. Boulanger, Experimental demonstration of a pure third-order optical parametric downconversion process, Opt. Lett. 29, 2794 (2004).

[9] M. V. Chekhova, O. A. Ivanova, V. Berardi, and A. Garuccio, Spectral properties of three-photon entangled states generated via three-photon parametric down-conversion in a $\chi^{(3)}$ medium, Phys. Rev. A 72, 023818 (2005).

[10] F. Gravier and B. Boulanger, Triple-photon generation: comparison between theory and experiment, J. Opt. Soc. Am. B 25, 98 (2008).

[11] M. Corona, K. Garay-Palmett, and A. B. U'Ren, Experimental proposal for the generation of entangled photon triplets by thirdorder spontaneous parametric downconversion in optical fibers, Opt. Lett. 36, 190 (2011).

[12] M. G. Moebius, F. Herrera, S. Griesse-Nascimento, O. Reshef, C. C. Evans, G. G. Guerreschi, A. Aspuru-Guzik, and E. Mazur, Efficient photon triplet generation in integrated nanophotonic waveguides, Opt. Express 24, 9932 (2016).

[13] A. Cavanna, F. Just, X. Jiang, G. Leuchs, M. V. Chekhova, P. S. Russell, and N. Y. Joly, Hybrid photonic-crystal fiber for singlemode phase matched generation of third harmonic and photon triplets, Optica 3, 952 (2016).

[14] E. A. R. González, A. Borne, B. Boulanger, J. A. Levenson, and K. Bencheikh, Continuous-Variable Triple-Photon States Quantum Entanglement, Phys. Rev. Lett. 120, 043601 (2018).

[15] M. Akbari and A. Kalachev, Third-order spontaneous parametric down-conversion in a ring microcavity, Laser Phys. Lett. 13, 115204 (2016).

[16] C. W. S. Chang, C. Sabín, P. Forn-Díaz, F. Quijandría, A. M. Vadiraj, I. Nsanzineza, G. Johansson, and C. M. Wilson, Observation of Three-Photon Spontaneous Parametric Down- 
conversion in a Superconducting Parametric Cavity, Phys. Rev. X 10, 011011 (2020).

[17] H. Hübel, D. R. Hamel, A. Fedrizzi, S. Ramelow, K. J. Resch, and T. Jennewein, Direct generation of photon triplets using cascaded photon-pair sources, Nature (London) 466, 601 (2010).

[18] T. Guerreiro, A. Martin, B. Sanguinetti, J. S. Pelc, C. Langrock, M. M. Fejer, N. Gisin, H. Zbinden, N. Sangouard, and R. T. Thew, Nonlinear Interaction between Single Photons, Phys. Rev. Lett. 113, 173601 (2014).

[19] C. Okoth, A. Cavanna, N. Y. Joly, and M. V. Chekhova, Seeded and unseeded high-order parametric down-conversion, Phys. Rev. A 99, 043809 (2019).

[20] M. Liscidini and J. E. Sipe, Stimulated Emission Tomography, Phys. Rev. Lett. 111, 193602 (2013).

[21] G. Agrawal, Nonlinear Fiber Optics (Academic, New York, 2012).

[22] $F_{n}$ are vectors but we omit vector notation for simplicity.

[23] J. Hammer, A. Cavanna, R. Pennetta, M. V. Chekhova, P. S. Russell, and N. Y. Joly, Dispersion tuning in sub-micron tapers for third-harmonic and photon triplet generation, Opt. Lett. 43, 2320 (2018).

[24] T. A. Birks, G. J. Pearce, and D. M. Bird, Approximate band structure calculation for photonic bandgap fibres, Opt. Express 14, 9483 (2006).

[25] A. Cavanna, F. Just, P. R. Sharapova, M. Taheri, G. Leuchs, and M. V. Chekhova, Tunable optical parametric generator based on the pump spatial walk-off, Opt. Lett. 41, 646 (2016).

[26] N. L. Boling, A. J. Glass, and A. Owyoung, Empirical relationship for predicting nonlinear refractive index changes index changes in optical solids, IEEE J. Quantum Electron. 14, 601 (1978).

[27] C. Brée, A. Demircan, and G. Steinmeyer, Method for computing the nonlinear refractive index via keldysh theory, IEEE J. Quantum Electron. 46, 433 (2010).
[28] A. D. Pryamikov, A. S. Biriukov, A. F. Kosolapov, V. G. Plotnichenko, S. L. Semjonov, and E. M. Dianov, Demonstration of a waveguide regime for a silica hollow-core microstructured optical fiber with a negative curvature of the core boundary in the spectral region $>3.5 \mu \mathrm{m}$, Opt. Express 19, 1441 (2011).

[29] C. Wei, R. J. Weiblen, C. R. Menyuk, and J. Hu, Negative curvature fibers, Adv. Opt. Photon. 9, 504 (2017).

[30] P. Uebel, M. C. Günendi, M. H. Frosz, G. Ahmed, N. N. Edavalath, J.-M. Ménard, and P. S. J. Russell, Broadband robustly single-mode hollow-core pcf by resonant filtering of higher-order modes, Opt. Lett. 41, 1961 (2016).

[31] J. Nold, P. Hölzer, N. Y. Joly, G. K. L. Wong, A. Nazarkin, A. Podlipensky, M. Scharrer, and P. S. Russell, Pressurecontrolled phase matching to third harmonic in ar-filled hollowcore photonic crystal fiber, Opt. Lett. 35, 2922 (2010).

[32] J.-L. Archambault, R. J. Black, S. Lacroix, and J. Bures, Loss calculations for antiresonant waveguides, J. Lightwave Technol. 11, 416 (1993).

[33] M. Zeisberger and M. A. Schmidt, Analytic model for the complex effective index of the leaky modes of tube-type antiresonant hollow core fibers, Sci. Rep. 7, 11761 (2017).

[34] N. Borshchevskaia, K. Katamadze, S. Kulik, S. Klyamkin, S. Chuvikov, A. Sysolyatin, S. Tsvetkov, and M. Fedorov, Luminescence in germania-silica fibers in a $1-2 \mu \mathrm{m}$ region, Opt. Lett. 42, 2874 (2017).

[35] H. J. Lehmeier, W. Leupacher, and A. Penzkofer, Nonresonant third order hyperpolarizability of rare gases and $\mathrm{n} 2$ determined by third harmonic generation, Opt. Commun. 56, 67 (1985).

[36] T. Birks and Y. W. Li, The shape of fiber tapers, J. Lightwave Technol. 10, 432 (1992).

[37] A. W. Snyder and J. Love, Optical Waveguide Theory (Springer, New York, 1983).

[38] J. D. Love and W. M. Henry, Quantifying loss minimisation in single-mode fibre tapers, Electron. Lett. 22, 912 (1986). 